\title{
VivaxGEN Tutorial
}

Release

Hidayat Trimarsanto, Sarah Auburn

Oct 13, 2016 

1 UPLOADING YOUR DATA 1

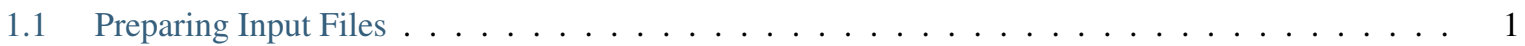

1.2 Creating a New Batch . . . . . . . . . . . . . . . . . . . . . . 2

1.3 Adding Sample Data . . . . . . . . . . . . . . . . . . . . . . . 2

1.4 Adding FSA Zip File . . . . . . . . . . . . . . . . . . . . . . . . . . . 3

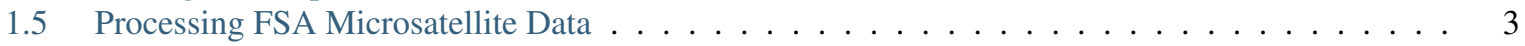

1.6 Assessing Fragment Analysis Results . . . . . . . . . . . . . . . . . 3

2 MICROSATELLITE DATA CLEANING $\mathbf{5}$

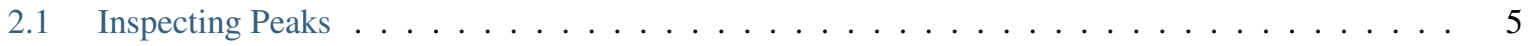

2.2 Inspecting Traces and Re-annotating Peaks $\ldots \ldots \ldots \ldots \ldots \ldots \ldots$

2.3 Inspecting Alleles with Lower Absolute Threshold or Relative Threshold . . . . . . . . . . . . 9

3 DATA ANALYSIS 11

3.1 Inspecting Sample Metadata . . . . . . . . . . . . . . . . . . . . . . 11

3.2 PCoA Cluster Plot . . . . . . . . . . . . . . . . . . . . . . . . . 13

3.3 PCoA Cluster Plot with Sample Grouping by Spatial Differentiation . . . . . . . . . . . . . . . 14

3.4 MCA Cluster Plot with Multiple Batches . . . . . . . . . . . . . . . . . . . . . . . . 15

4 DATA ANALYSIS WITH CUSTOM QUERY 17

4.1 Using the Custom Query Form . . . . . . . . . . . . . . . . . . . . . . . . 17

4.2 Performing Principal Coordinate Analysis using a Custom Query . . . . . . . . . . . . . . . 19

5 DATA ANALYSIS WITH YAML FORMAT 21

5.1 A Glance of YAML Format . . . . . . . . . . . . . . . . . . . . . . . . . 21

5.2 Using YAML Query . . . . . . . . . . . . . . . . . . . . . . 22

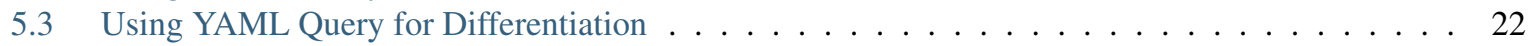




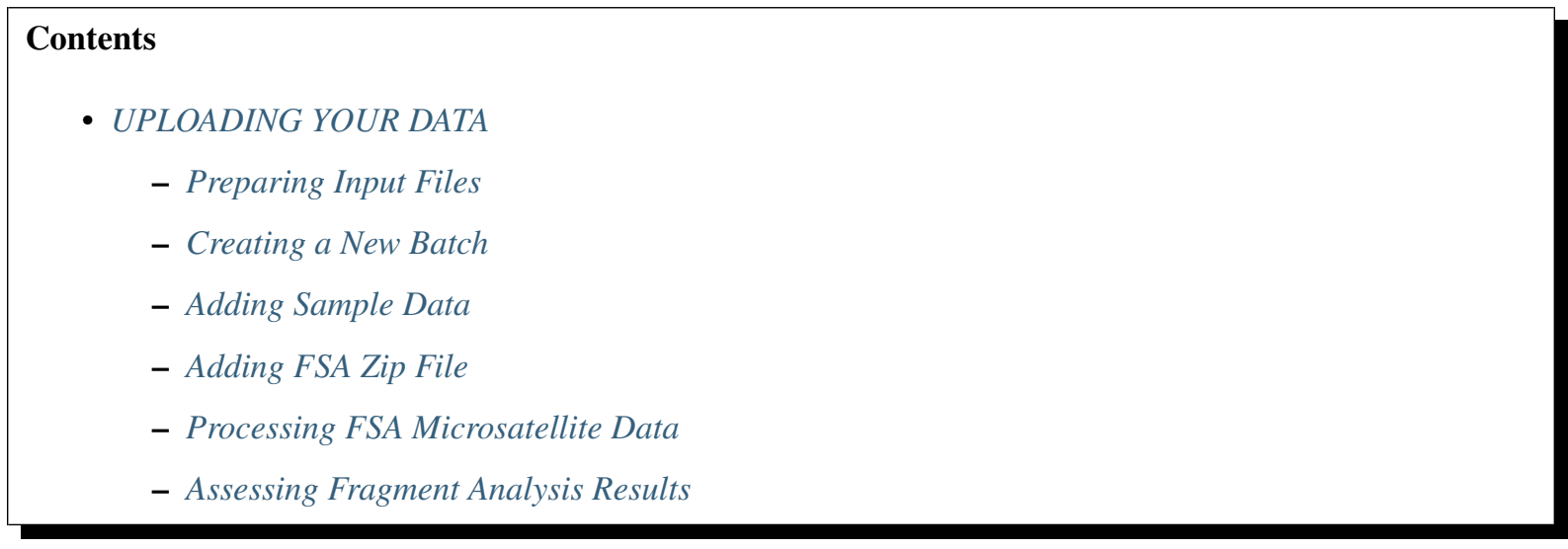

This tutorial provides step-by-step directions on how to prepare and upload your FSA files and metadata to the VivaxGEN platform, with an accompanying example Plasmodium vivax microsatellite dataset. A more detailed guide for data preparation and uploading can be found in the Guide: Data preparation and Uploading. Note, it is assumed that users have a good understanding of the general concepts of microsatellite-based genotyping.

\subsection{Preparing Input Files}

The example datasets, available as data-01.zip, consist of three files:

1. sampleinfo.txt - A tab-delimited file containing sample metadata.

2. fsa.zip - A zipped file containing all microsatellite data in FSA-formatted files.

3. assayinfo.txt - A tab-delimited file containing FSA metadata

The sampleinfo.txt and assayinfo.txt files can be opened for inspection using any software capable of reading text files or spreadsheet-based softwares such as Microsoft Excel or LibreOffice Calc.

More detailed information on the file format can be found here: Guide - File Format.

When preparing your own input files, any name can be used for each of the three files, but the file formatting must be strictly adhered to.

Please note that the system is primarily set up to accept data from Plasmodium vivax. If data is available for other Plasmodium Spp, this can be accommodated using the same input file formatting as long as the relevant fields are filled appropriately. It is strongly recommended to use different batches for different species as downstream analytical processes will require separation of the different species. 


\subsection{Creating a New Batch}

Before uploading the example datasets, you will need to create a new batch (unless you plan to add data to an existing batch). A batch is essentially a collection of samples and associated molecular data which the user intends to analyse together (i.e. from the same study).

Log in to VivaxGEN using the guest or private account details. To establish a private account, with private username and passowrd, you will need to send an email request to the systems administrator at anto@eijkman.go.id.

Once logged in to VivaxGEN, select Manage data or Browse $\gg$ Batch from the navigation menu. A list of existing batch names, including publically available batches, will be displayed (these names cannot be used for new batches). To create a new batch, select New batch. To add data or update an existing batch, you will need to select the intended batch name instead.

For new batches, you will be provided with a form with details to fill in as listed below. Compulsory fields are stated.

Batch code Compulsory field. A unique (i.e. not already present in the database) string that identifies your batch. Allowed characters for the string are alphanumerics, dash or underscore. The maximum length for batch code is 16 characters. Do not use any spaces. Best practise is to use a combination of country identification, species and year, such as IDPV2015 for Indonesian $P$ vivax in year 2015.

Description Optional but recommended field. A brief description outlining the nature of the samples and the study for which the data was generated.

Primary group Compulsory field. A string indicating the name of the group or organization providing the data, i.e. the data owner. For this tutorial, set as DEMOGROUP.

Assay provider group Compulsory field. A string indicating the name of the organization where the assays were run. For the accompanying example datasets in this tutorial, set as MACROGEN.

Batch for bins setting Compulsory field. This is the batch code that will be used as the reference for bin settings. This option allows different batches to have different bins settings and parameters (for examples, bins for LIZ600 and bins for LIZ500). For now, just use default.

Species Compulsory field. A string indicating the Plasmodium Spp. The system currently supports $\mathrm{Pv}$ and Pf assays. For this tutorial, set as Pv. Important: by setting species, the system will assume that any markers without explicit species code mentioned in any input files are markers for this intended species, unless the species is explicitly stated. For example, marker MS16 will be assumed as pv/MS16.

Remarks Optional field. An optional field for any information regarding this batches, further detailed description on the samples or the study that may be helpful for those who are going to use the data in this batch.

Once you have completed the forms, select Save. You will then be directed to the batch view page, where you can manage the given batch.

\subsection{Adding Sample Data}

On the Batch view, select Choose file or Browse next to Sample Info file, and select the sample info file (sampleinfo.tab in the tutorial). Then select Upload to temporarily save the sample information file in the VivaxGEN platform. Select Verify to check if the sample information file contains any errors. In case of errors, a message detailing the error lines will be returned. Correct any errors and re-upload the sample information file. Ensure that the appropriate sample submission option is checked - for the tutorial, leave as default (Add new samples and update existing samples). Select Proceed to save the sample information file in the VivaxGEN platform. On the Uploading Report view, if the sample information was uploaded successfully, select Continue to return to the Batch view. 


\subsection{Adding FSA Zip File}

From the Batch view, under FSA Bulk Uploading, select Start upload session. In the FSA Bulk Upload Manager view, click on Select and upload FSA archive file and select the fsa zip file (fsa.zip in the tutorial) to upload the FSA files to VivaxGEN. Once the uploading is finished, select Continue to verify the uploaded archive file to check that the files were uploaded correctly. In case of errors, a message detailing the error lines will be returned. Correct any errors and re-upload the fsa zip file.

If there are no errors, click on Continue to upload FSA info file (CSV or tab-delimited) and select the assay information file (assayinfo.tab in the tutorial) to the VivaxGEN platform. Select Continue to verify FSA info file to check that the file was uploaded correctly. In case of errors, a message detailing the error lines will be returned. Correct any errors and re-upload the assayinfo file by selecting Change/replace the uploaded FSA info file.

If there are no errors, select Continue to process FSA files to save each of the FSA files to the VivaxGEN platform. This process may take a few minutes. Once uploading is finished, select Continue to return to the Batch view. In case FSA Bulk Uploading is interrupted at any point, you can return to the incomplete session by selecting List pending sessions and then selecting the corresponding session.

\subsection{Processing FSA Microsatellite Data}

Once the FSA files have been saved, fragment analysis (see the manuscript for further details on this process) must be undertaken. From the Batch view, select Start FSA FA Manager, and then select Process FSA. Note that this is a lengthy task, with the time required depending on the number of FSA files/assays submitted, and how "noisy" the traces are. Please also note that you can continue other tasks in VivaxGEN in parallel or log out of the platform without impeding the fragment analysis processing. If you choose to log out during this processing step, on returning to VivaxGEN, you can navigate back to the FSA FA manager view to inspect progress. Once assay processing is finshed, select Continue, and then select Browse FSA files to starting inspecting individual FSA files as described in step 6 (or batch name to return to the batch view). Note that further filtering of alleles by absolute and relative allele peak intensity are provided in the analysis tools.

\subsection{Assessing Fragment Analysis Results}

Once the fragment analysis process has finished, it is recommended to assess the results of the processing. On the FSA FA manager view, select Browse FSA files to open a new page showing the list of the uploaded FSA files together with their parameter results.

The details of the parameters are outlined below:

FSA Filename The name of the FSA file

Sample Code The sample code for the corresponding FSA file

Panel The panel used for the corresponding FSA file

Score The quality of ladder peaks of the FSA file, from 0.00 to 1.00 (highest score).

RSS The Residual Sum of Squares of the ladder peaks against the regression line. Lower RSS value $(<50.00)$ indicates higher quality of the FSA file.

Proctime The time taken for the system to process the FSA file in miliseconds. Higher processing time usually indicates that the FSA file is noisy.

To inspect individual FSA files, select the corresponding FSA filename (good practise is by right-clicking the mouse button to open a new tab) which will open the FSA viewer. Individual peaks (alleles) can be manually edited (or re-annotated) by selecting the Edit link in the corresponding peak/allele tables. Once the allele-calling has been finalized, several population genetic analyses can be performed using a suite of tools available under Analyze in the navigation menu (see Tutorial 2). Note that further filtering of alleles by absolute and relative allele peak intensity thresholds are provided in the analysis tools. 
If there are any errors at any of steps in the process that cannot be resolved, please contact the systems administrator at anto@eijkman.go.id. 


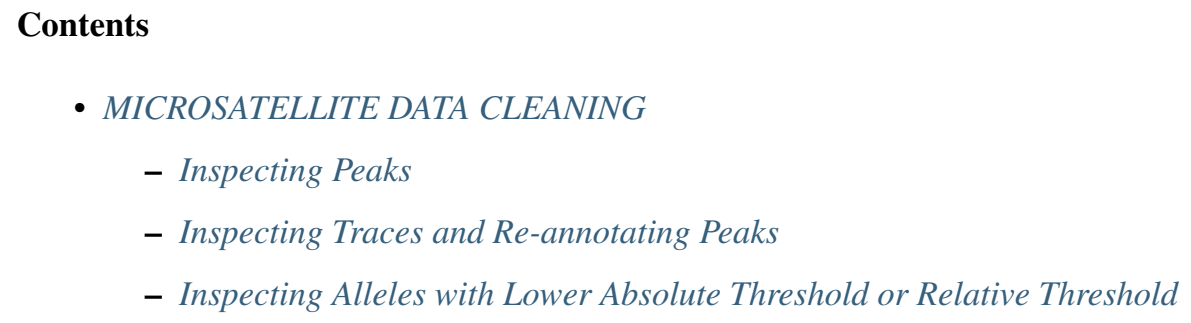

This tutorial provides step-by-step instructions on how to perform data cleaning to exclude peaks such background noise, artefacts or stutter. The fragment analysis process in VivaxGEN provides the first layer of data cleaning from the raw FSA files. Several algorithms, described further in the Integrated Fragment Analysis Tools section of the manuscript, perform automated annotation of peaks as either bin peaks (real alleles) or non-binned peaks such as stutter and artefacts, which are excluded from further data analysis. However, some peaks may not be annotated correctly by the algorithms, especially in challenging FSA files such as those containing low intensity peaks or extensive background noise. The VivaxGEN platform therefore provides tools for inspecting the raw electropherogram traces to cross-check the automated annotations, and to manually re-annotate peak definitions where needed. As the tutorial requires uncleaned data, you will need to use the new sample batch that you created and processed in Tutorial 1.

\subsection{Inspecting Peaks}

We will start by inspecting the summary of all genotyped peaks (i.e. all peaks defined as true alleles by the automated fragment analysis algorithms) in the data set using the Genotype Summary tool. Select the Genotype Summary entry from the Analyze drop-down menu. This will take you to a web page with a form for selecting the sample batch and markers, and for sample and marker filtering according to a number of parameters described further in the Tools for Allele and Sample Filtering section of the manuscript. A snapshot of the form is provided in the figure below. 


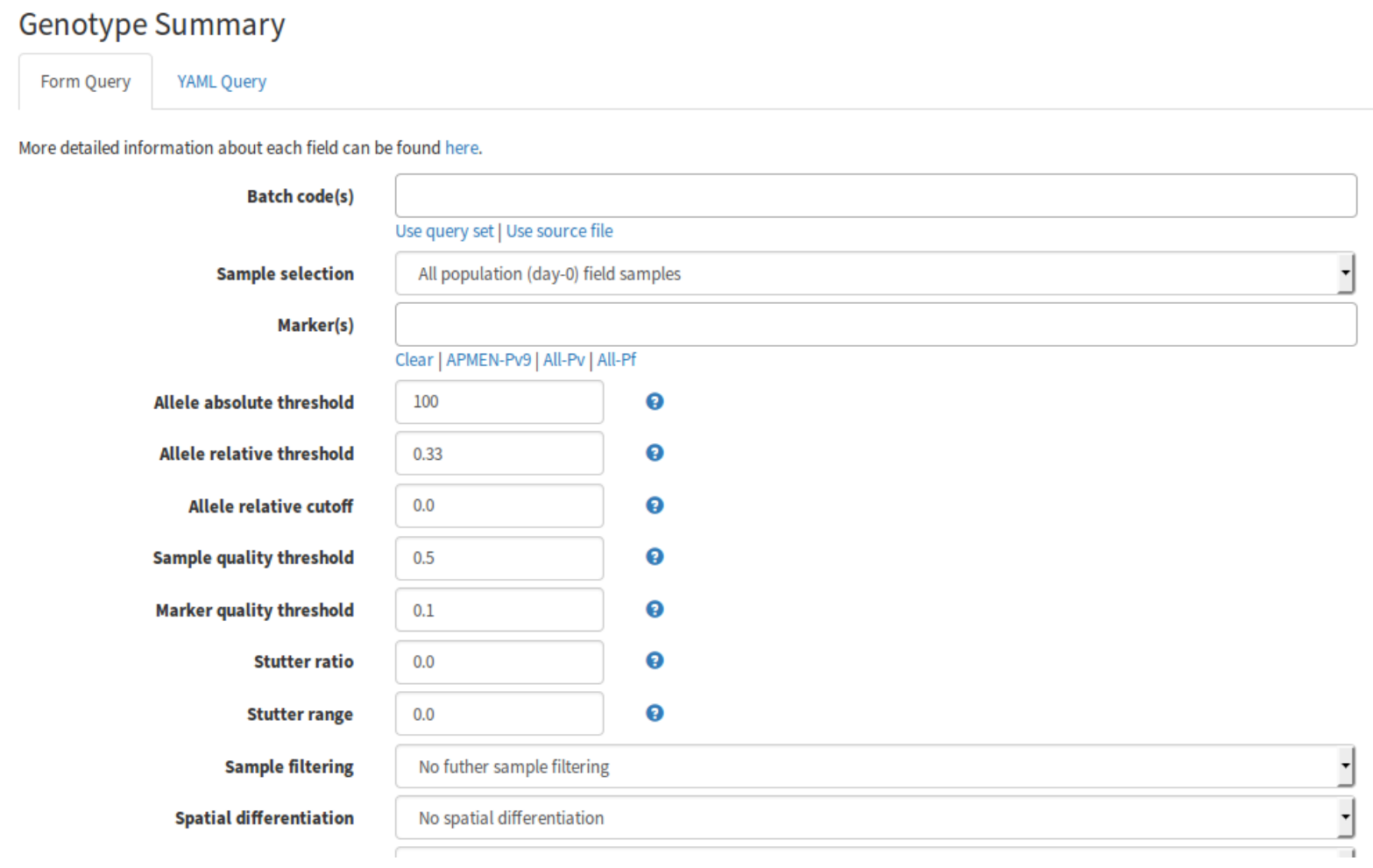

In the Batch code(s) field, select the new, uncleaned batch that you created in Tutorial 1. The samples in the Tutorial 1 batch were genotyped at the 9 APMEN P. vivax markers (see reference ) - click on the APMEN-Pv9 link to select these markers. This will populate the Marker(s) field with the appropriate markers. In this step of the tutorial, we will leave all other parameters as default. Note that the default value of the Allele absolute threshold is set to 100 relative fluorescence units (RFU) i.e. all peaks with RFU less than 100 will be excluded even if they were binned by the automated algorithm. Select Execute to perform the analysis. Once complete, a Genotype Summary report will be provided as illustrated in the figure below.

\section{Genotype Summary}

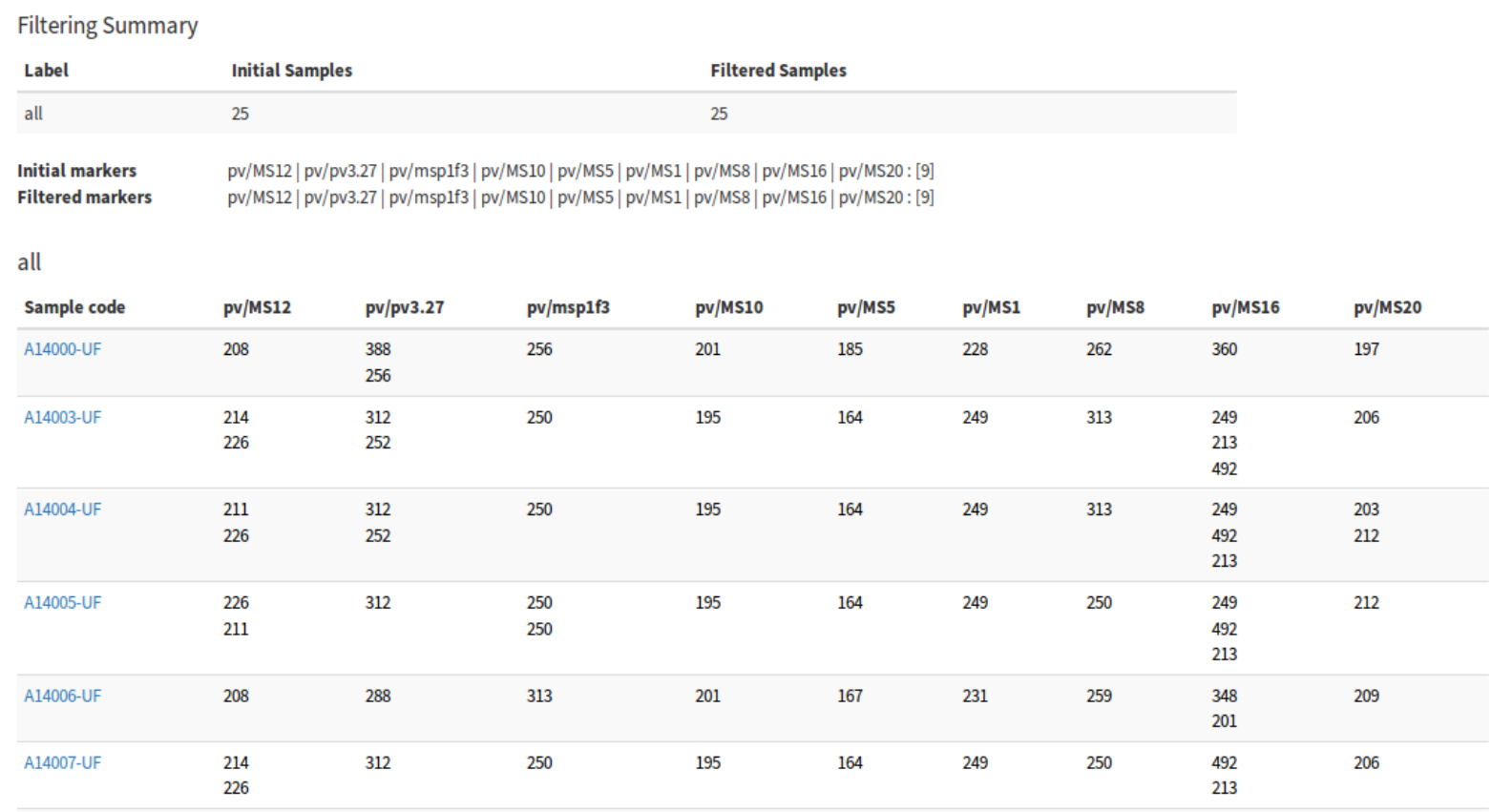

If we look closely at the results, we will see that there are sample/marker combinations that have multiple alleles. For example, there are multiple alleles in many of the samples at markers MS12 and MS16. The multiple alleles within sample/marker combinations are sorted by their heights. In some cases, all of the alleles may be real, reflecting multiple clone infections in which different clones have different alleles. However, in some cases, one 
or more of the alleles may reflect peaks from noise, stutter, overlap or other artefacts that were not correctly annotated by the automated fragment analysis algorithms. Please note that the VivaxGEN platform is under constant development to improve features such as the fragment analysis peak annotation and the results of the automated fragment analysis peak annotation may differ slightly between different versions of the platformwhen this tutorial was written.

\subsection{Inspecting Traces and Re-annotating Peaks}

To aid judgment of whether a given peak reflects a true allele, we need to inspect the original electropherogram trace in the FSA file. We can do this using the FSA Viewer, which can be accessed directly by clicking on an allele of interest.

\begin{tabular}{|c|c|c|c|c|c|c|c|c|c|}
\hline UFA-01-MN & 223 & 304 & 262 & 174 & $\begin{array}{l}197 \\
224\end{array}$ & $\begin{array}{l}216 \\
234\end{array}$ & 214 & 369 & $\begin{array}{l}224 \\
203\end{array}$ \\
\hline UFA-02-MN & 208 & 316 & 262 & 222 & 185 & 228 & 280 & 342 & $\begin{array}{l}197 \\
224\end{array}$ \\
\hline
\end{tabular}

As an example, locate sample UFA-01-MN as indicated above, and open the trace view for allele 224 of MS5 marker by clicking on 224 using the right mouse button and selecting open link in new tab. The trace view should look similar to the figure below.

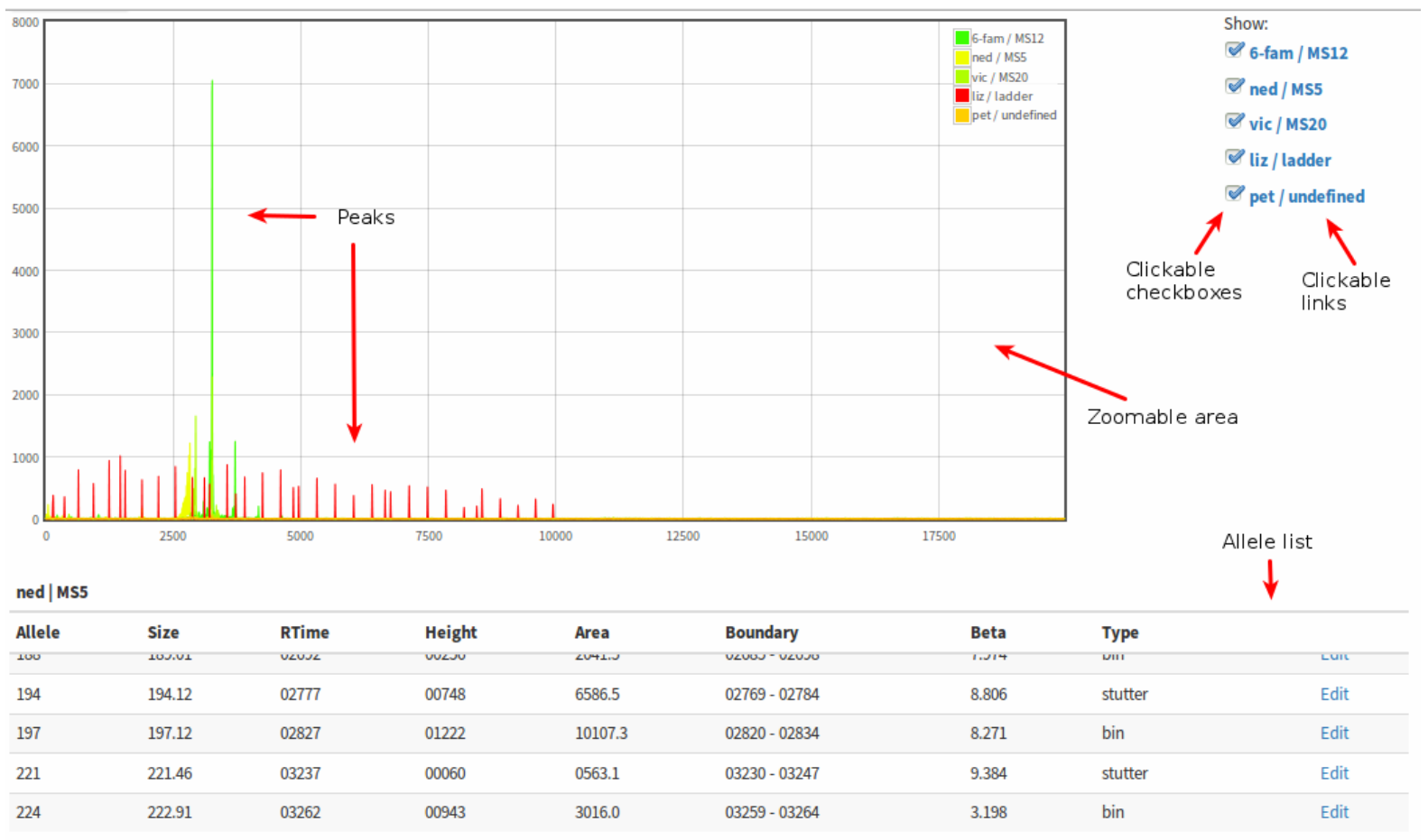

The view consists of the electropherogram trace from the FSA file and a summary of allele peaks and associated annotations below. We can zoom in on the trace by selecting an area using the mouse. To the right of the trace is a panel containing checkboxes which allow the user to turn specific markers/dyes on or off. Clicking on the dye or marker name will scroll the allele list to the designated dye/marker.

As an example, turn off all dyes/markers except MS5 and zoom in on the trace to the area around 2400 - 3400 retention time to generate a view similar to the figure below. 


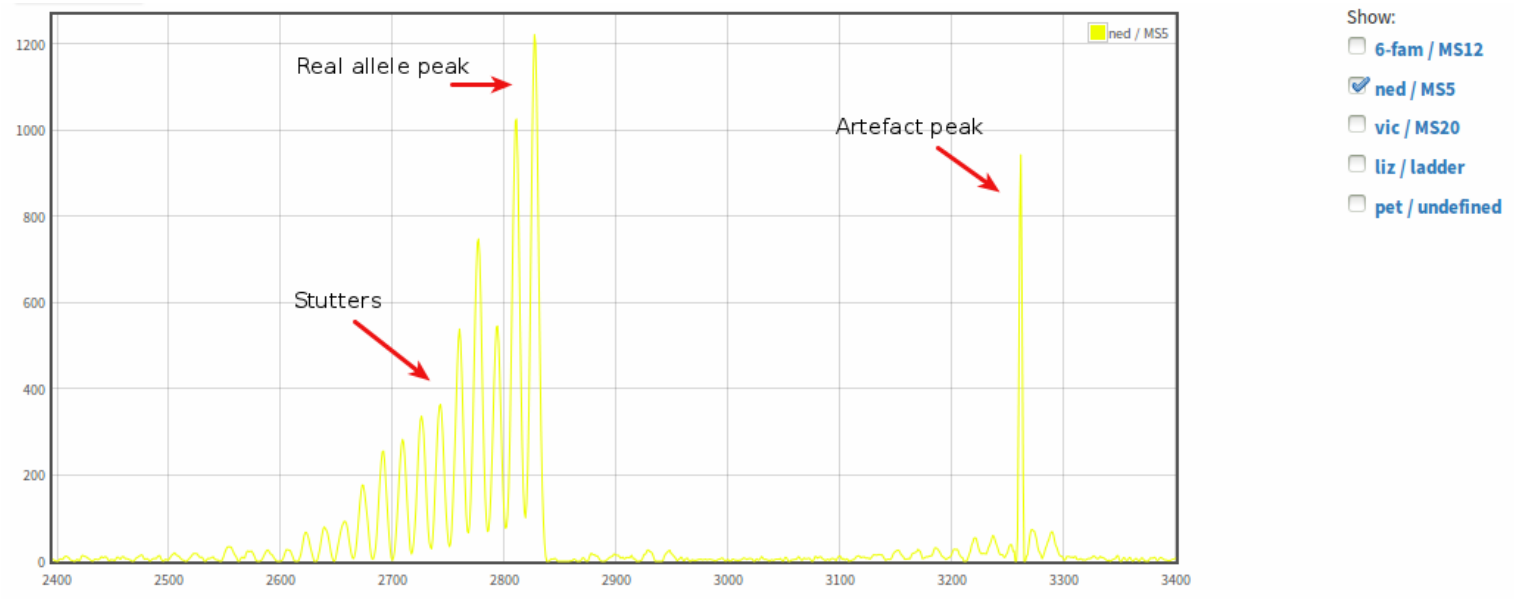

\begin{tabular}{|c|c|c|c|c|c|c|c|c|}
\hline Allele & Size & RTime & Height & Area & Boundary & Beta & Type & \\
\hline 197 & 197.12 & 02827 & 01222 & 10107.3 & $02820-02834$ & 8.271 & bin & Edit \\
\hline 224 & 222.91 & 03262 & 00943 & 3016.0 & $03259-03264$ & 3.198 & bin & Edit \\
\hline
\end{tabular}

Note that the peak underlying allele 224 at retention time $\sim 3262$ has a very narrow base uncharacteristic of the alleles for this marker. The beta value (height divided by width) of this peak is 3.2 , whereas most real alleles have beta of 5-10. Moreover, the peak is not accompanied by small stutter peaks or widening areas close to baseline that usually form around real allele peaks. If we switch the other markers (MS12 and MS20) back on and zoom back into the region, we can see that the MS5 224 allele is overlapped by a large MS12 peak and is therefore likely to be an overlap peak and not a true allele (as illustrated below).

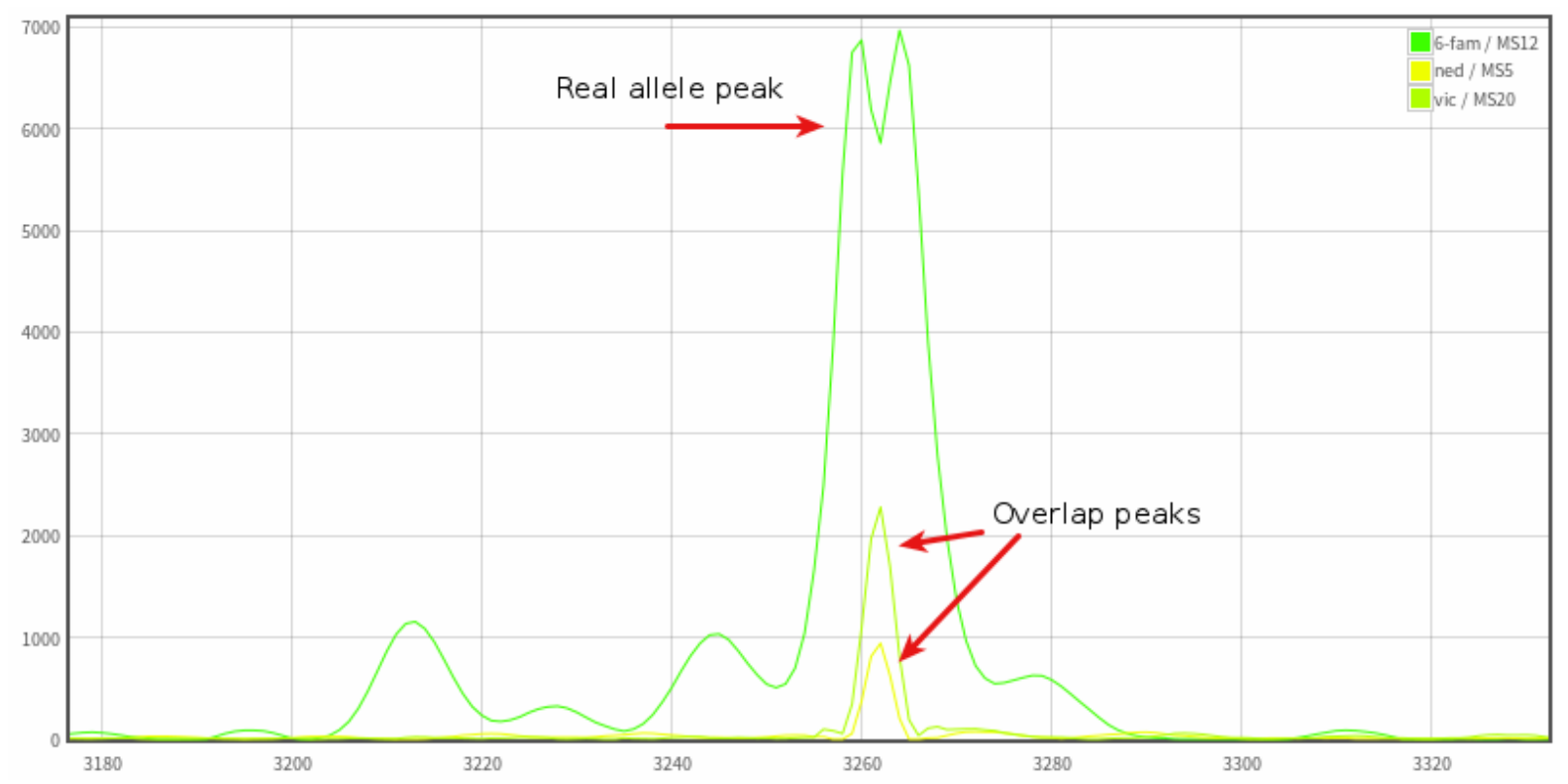

We can manually re-annotate this peak as overlap peak (or any other annotation other than bin) to exclude it from further analyses. To do so, click on the Edit link at the row of allele 224, which will bring up a pop up window as illustrated below. 


\section{Edit Allele}

Marker

pv/MS5
Size

222.91
RTime

3262
Height

943

Bin

224

Type

bin

Update allele

Reset

From the pop-up window, we can change Type field from bin to overlap (or any other annotation other than bin) and then click Update allele. The updated annotation will be saved. We can then return to the Genotype Summary tab (or window), and click on Resubmit analysis on the top left of the page to get the updated result. The allele 224 at MS5 marker from sample UFA-01-MN should not appear in the updated Genotype Summary.

\subsection{Inspecting Alleles with Lower Absolute Threshold or Relative Threshold}

In many cases, false allele peaks may be excluded by adjusting the Allele absolute threshold and/or Allele relative threshold. In the previous steps, we observed our data at the default Allele absolute threshold of 100 RFU. To inspect the Genotype Summary at a lower threshold, we can change the value of the Allele absolute threshold and/or Allele relative threshold to lower values in the Genotype Summary form. Note that the public batches available in VivaxGEN have all been cleaned to Allele absolute threshold of 40 RFU. 
TUTORIAL 3

DATA ANALYSIS

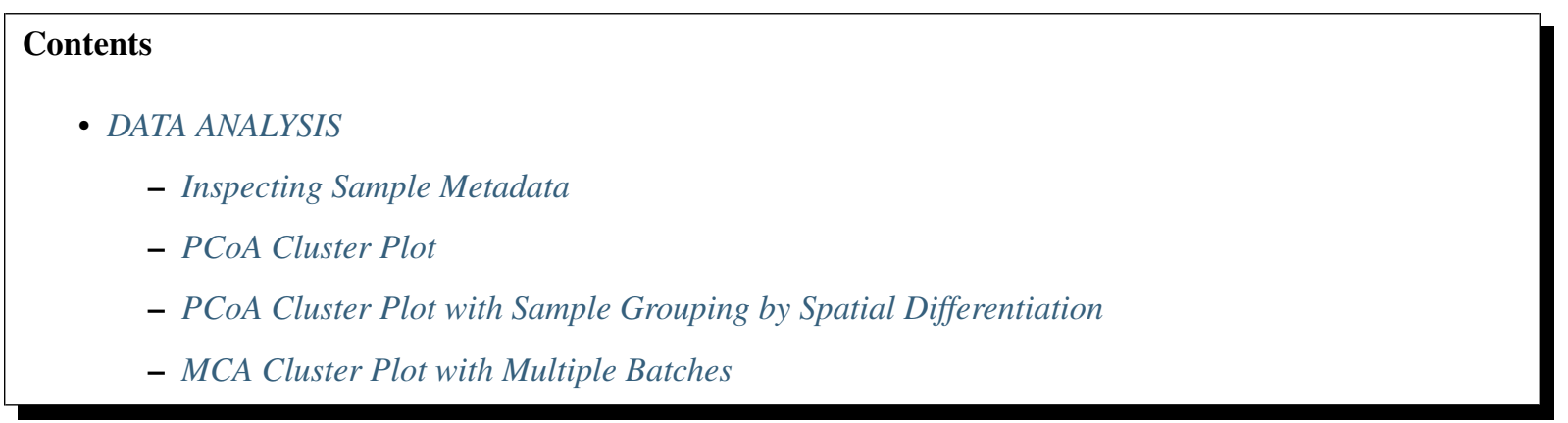

This tutorial provides step-by-step instructions on how to perform data analyses using the form-based web interface. The tutorial uses examples from the publically available batch BTPV, comprising microsatellite data on $P$. vivax isolates from patients in Bhutan (1).

\subsection{Inspecting Sample Metadata}

Before performing further analyses on batch BTPV, we will start by determining how many samples the batch has, and inspecting some features of the sample metadata. For this step, we will use the Sample summary analysis tool. Select the Sample summary entry from the Analyze drop-down menu, and a form similar to the figure below will appear. 


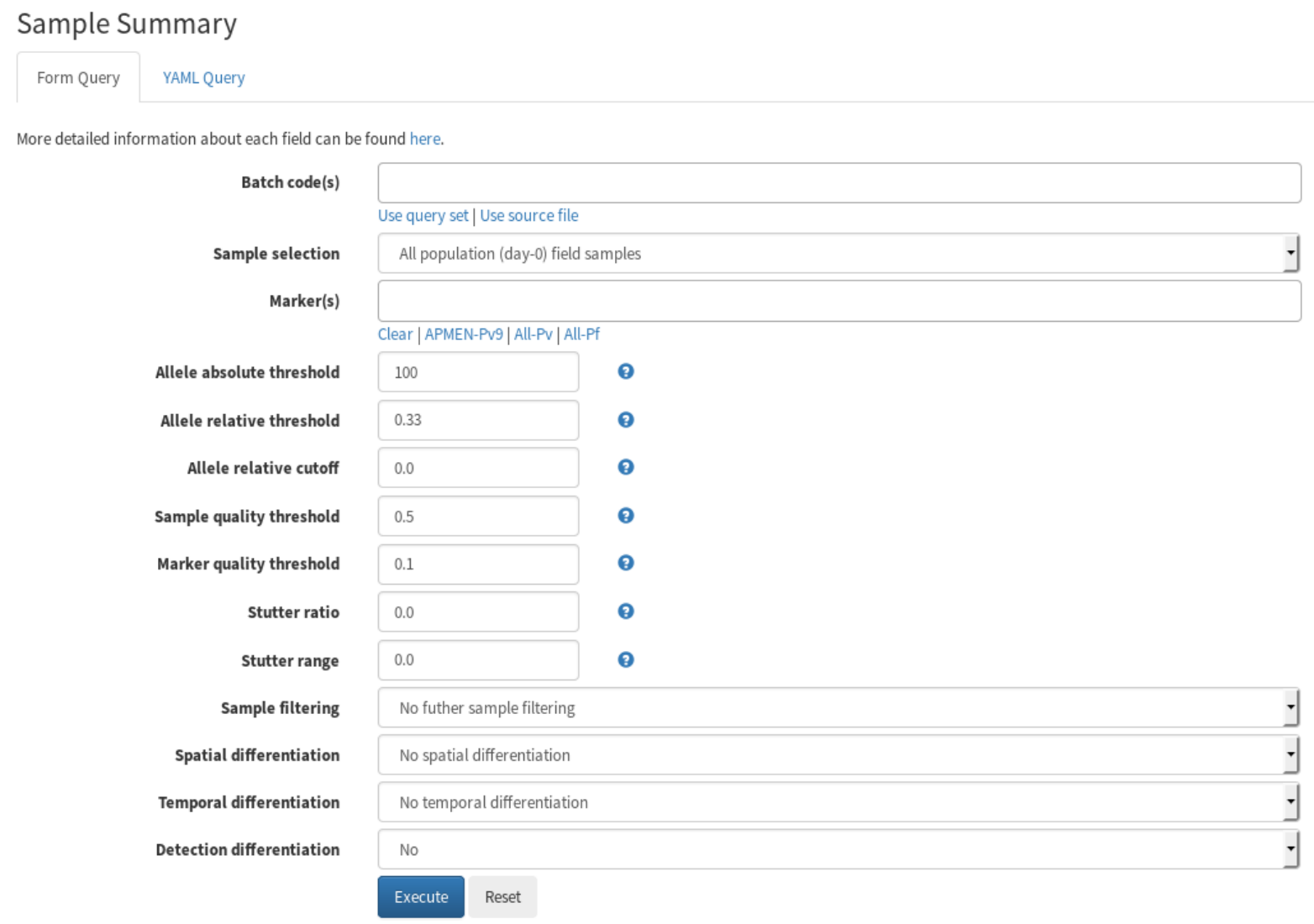

To use the BTPV batch, select BTPV from the Batch code(s) field. As we plan to analyze all independent samples in the batch (i.e. all day-0 samples), the Sample selection field should be left as default. The samples in the BTPV batch were genotyped at the 9 APMEN P. vivax markers (2), click on the APMEN-Pv9 link to select these markers. This will populate the Marker(s) field with the appropriate markers.

As detailed in the Tools for Allele and Sample Filtering section of the manuscript, samples and markers can be filtered according to a number of parameters. In this tutorial, we will use all of the default parameters. Once the parameters have been set, select Execute to perform the analysis. A snapshot of the report outlining the results of the sample summary query on the BTPV batch is illustrated in the figure below. 


\section{Sample/Metadata Summary Report}

\section{Filtering Summary}

\begin{tabular}{lll} 
Label & Initial Samples & Filtered Samples \\
\hline all & 28 & 28 \\
& \\
Initial markers & $\mathrm{pv} / \mathrm{MS} 12|\mathrm{pv} / \mathrm{pv} 3.27| \mathrm{pv} / \mathrm{msplf} 3|\mathrm{pv} / \mathrm{MS} 10| \mathrm{pv} / \mathrm{MS} 5|\mathrm{pv} / \mathrm{MS} 1| \mathrm{pv} / \mathrm{MS} 8|\mathrm{pv} / \mathrm{MS} 16| \mathrm{pv} / \mathrm{MS} 20:[9]$ \\
Filtered markers & $\mathrm{pv} / \mathrm{MS} 12|\mathrm{pv} / \mathrm{pv} 3.27| \mathrm{pv} / \mathrm{msplf3}|\mathrm{pv} / \mathrm{MS} 10| \mathrm{pv} / \mathrm{MS} 5|\mathrm{pv} / \mathrm{MS} 1| \mathrm{pv} / \mathrm{MS} 8|\mathrm{pv} / \mathrm{MS} 16| \mathrm{pv} / \mathrm{MS} 20:[9]$
\end{tabular}

all

\begin{tabular}{ll} 
string1 & N \\
\hline passive_detection & N \\
\hline True & 28 \\
\hline symptomatic_status & N \\
\hline False & 28 \\
\hline pcr_identity & N \\
\hline pv & 28 \\
\hline microscopy_identity & N \\
\hline pv & 28 \\
\hline blood_withdrawal & N \\
\hline venous & 28 \\
\hline imported_case & N \\
\hline & 28 \\
\hline
\end{tabular}

The following information is provided:

- There are 28 samples in total (Initial Samples) in batch BTPV.

- After filtering samples and markers according to the parameters set in the prior step, there are 28 samples remaining (Filtered Samples) in batch BTPV and that have been included in the analysis. As defined in the default parameters, the dataset on the 28 Filtered samples only comprises samples with genotype calls for at least $50 \%$ of the total markers (i.e. 5 of the 9 markers), as the default value of Sample quality threshold is 0.5. As the default Allele absolute threshold was set to 100, genotype calls were only provided for alleles with relative fluorescence unit $(\mathrm{RFU})>=100$.

- All 28 Filtered samples were collected by passive detection, without any symptomatic status, and had been identified as $P$. vivax by both PCR and microscopy. All blood samples were collected by venous withdrawal.

- The patient donors included 16 Indian nationals and 12 Bhutanese nationals. Three of the patients were female and 25 were male.

\subsection{PCoA Cluster Plot}

In this tutorial step, we will review the steps for performing Principal Coordinate Analysis (PCoA) in the BTPV batch as an example on how to apply one of the platform's suite of standard population genetic tools using the form-based web interface.

Select the Principle Coordinate Analysis (PCoA) entry from the Analyze drop-down menu. A form similar to that used for inspecting the sample metadata will be provided to enable sample and marker filtering as required. Note that the sample and marker filtering form is available for all analyses within the VivaxGEN platform. 
Fill the form as before:

- Use Batch code(s) BTPV

- Use Markers(s) APMEN-Pv9

- Leave all other fields as default

When the form has been filled, select Execute to run the analysis. The results output should provide a PCoA cluster plot similar to the figure below. This is the simplest form of analysis, with minimal annotation by metadata details such as spatial and temporal parameters.

\section{Principal Coordinate Analysis (PCoA) Result}

Filtering Summary

\begin{tabular}{llll} 
Label & Initial Samples & Filtered Samples & MLG Samples \\
\hline all & 28 & 28 & 28
\end{tabular}

Initial markers $\quad$ pv/MS12 1 pv/pv3.27|pv/msplf3 | pv/MS10 |pv/MS5 | pv/MS1 |pv/MS8 | pv/MS16|pv/MS20: [9]

Filtered markers $\quad$ pv/MS12 $|p v / p v 3.27| p v / m s p l f 3|p v / M S 10| p v / M S 5|p v / M S 1| p v / M S 8|p v / M S 16| p v / M S 20:[9]$

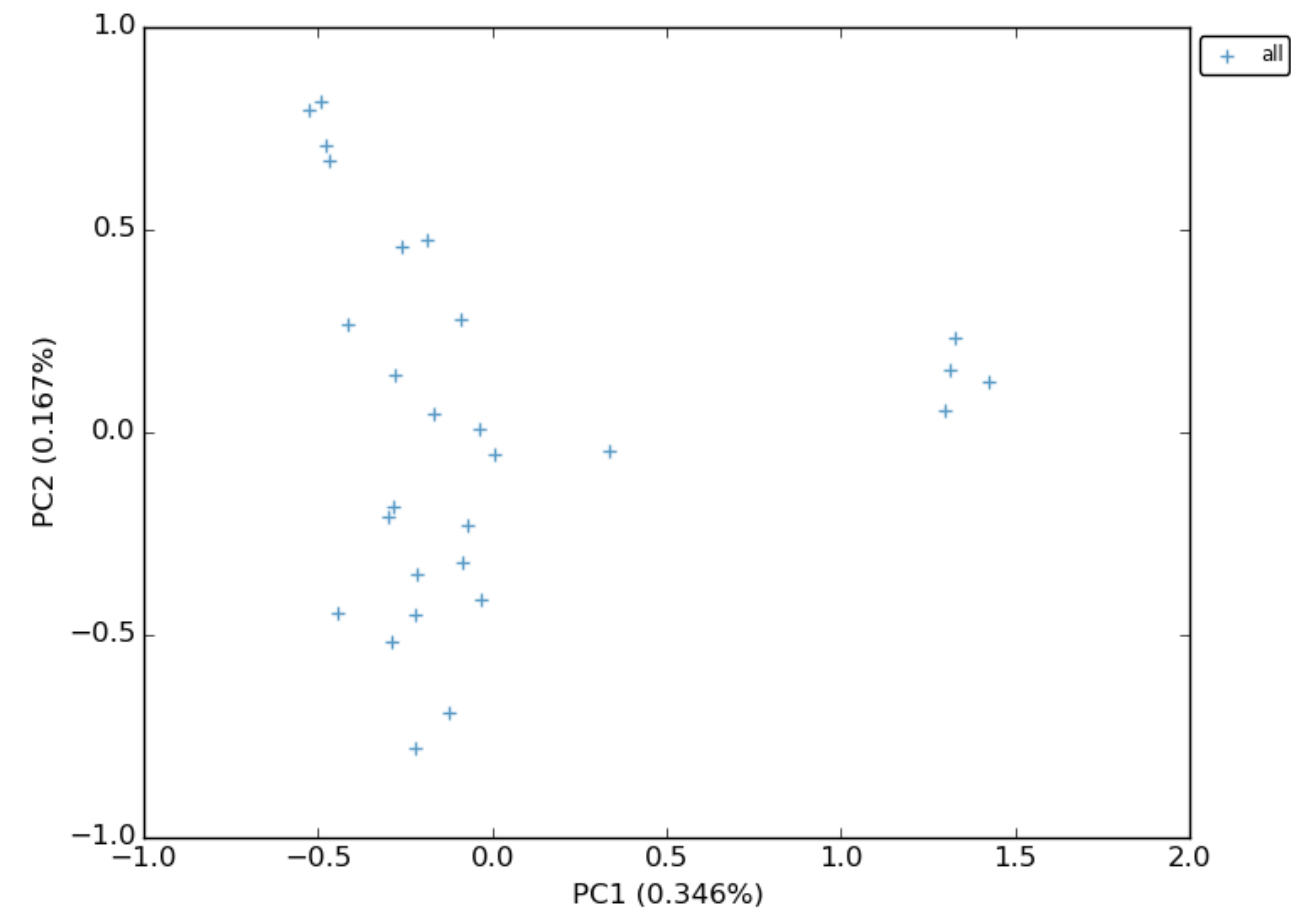

Note that PCoA can only be performed on samples with genotype calls for all markers selected (i.e. with complete multi-locus genotypes - MLGs). The Filtering Summary in the results output provides sample numbers for Initial Samples, Filtered Samples, and samples with complete MLGs (MLG Samples).

\subsection{PCoA Cluster Plot with Sample Grouping by Spatial Differenti- ation}

In this tutorial step, we will further explore the PCoA cluster plot in batch BTPV by overlaying more details on spatial differentiation. Return to the Principle Coordinate Analysis (PCoA) entry and use the same parameters as in step 2 except for the Spatial differentiation field - here, select 1st Administration level. When the form has been filled, select Execute to run the analysis. As illustrated in the figure below, a similar cluster plot to step 2 should be produced but with annotation on spatial differentiation at the 1st Administrative level. With the extra colour-coded spatial annotation, we can now see that the 4 samples which separated from the others on PC1 were all collected from Sarpang District. 


\begin{tabular}{llll} 
Label & Initial Samples & Filtered Samples & MLG Samples \\
\hline all | bhutan / gelephu & 1 & 1 & 1 \\
\hline all | bhutan / pemagatshel & 1 & 1 & 1 \\
\hline all | bhutan / samdrupjongkhar & 1 & 1 & 1 \\
\hline all | bhutan / sarpang & 16 & 16 & 16 \\
\hline all | bhutan / trongsa & 2 & 2 & 2 \\
\hline all | bhutan / tsirang & 1 & 1 & 1
\end{tabular}

Initial markers $\quad$ pv/MS12 $\quad$ pv/pv3.27 $\mid$ pv/msplf3 | pv/MS10 | pv/MS5 |pv/MS1|pv/MS8 |pv/MS16 |pv/MS20: [9]

Filtered markers $\quad$ pv/MS12 $|p v / p v 3.27| p v / m s p 1 f 3|p v / M S 10| p v / M S 5|p v / M S 1| p v / M S 8|p v / M S 16| p v / M S 20:[9]$

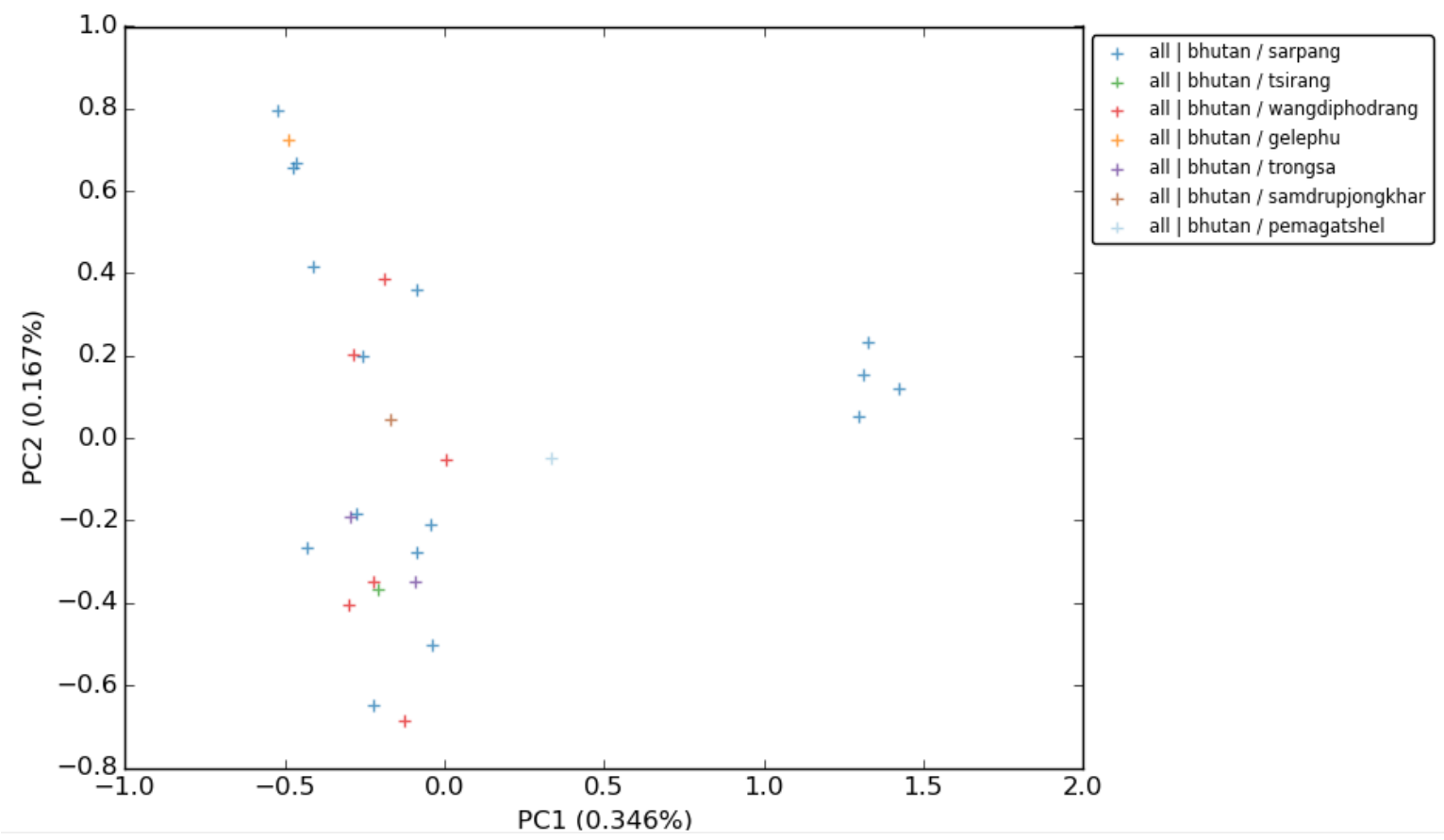

Note that the Filtering Summary now provides details on sample numbers by 1st Administration level.

\subsection{MCA Cluster Plot with Multiple Batches}

A more interesting question that can be inferred by cluster plot is comparing samples from different bigger regions such as countries, which can be done by using multiple batches from different countries. In this tutorial step, we will use Multiple Correspondence Analysis (MCA) which is another method of getting cluster plot.

Select the Multiple Correspondence Analysis (MCA) entry from the Analyze drop-down menu. A familiar form will be shown. However, instead of just selecting BTPV batch in the Batch code(s) field, add another batch by selecting ETPV batch which contains samples from Ethiopia. We also need to select Country level for Spatial differentiation field so that we will know which samples come which countries. The completed form will look similar to the following figure. 


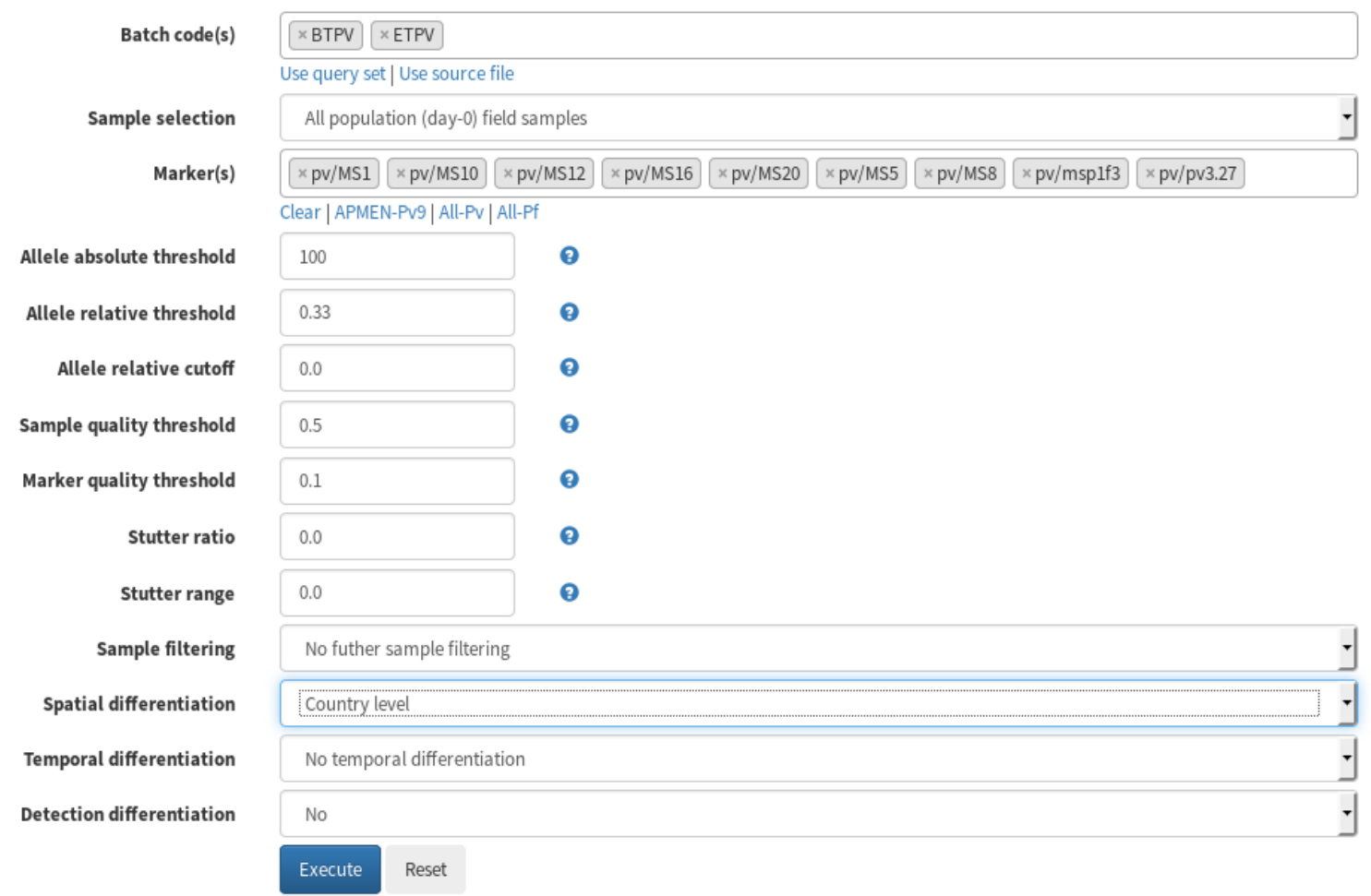

The reason we are confident in mixing BTPV and ETPV is that both batches were genotyped using APMEN $9 P$. vivax markers, and both used LIZ600 standard size.

Once the MCA analysis finishes, we will obtain a plot similar to the following figure.

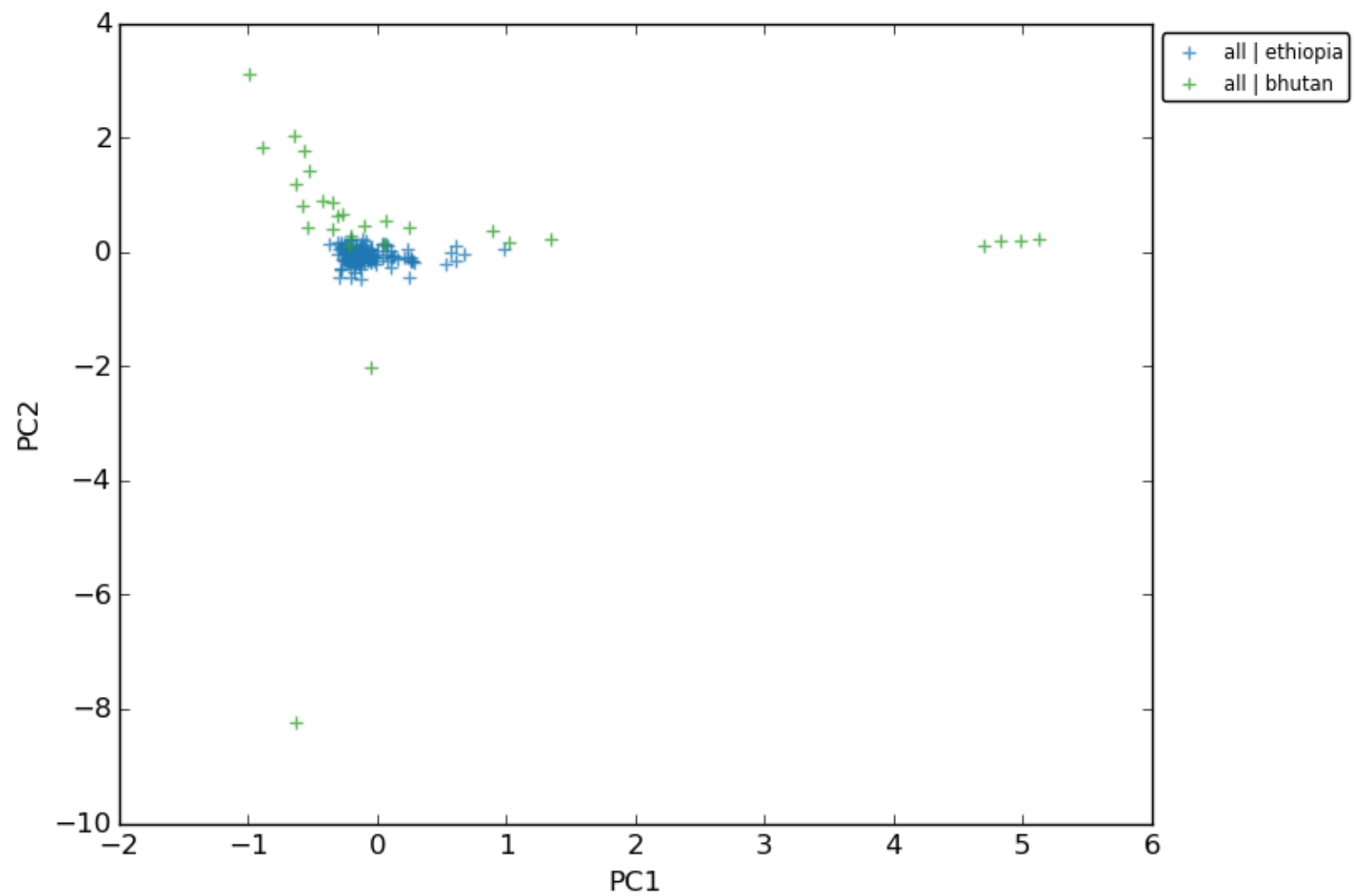

We can see from the result that the sample size from Ethiopia is much bigger than from Bhutan. Despite of this, the Bhutan cluster is more dispersed compared to the the much tight Ethiopia cluster. In molecular term, we can infer that the Bhutan samples have higher diversity relative to the Ethiopia samples. Please note that there are other methods that can be used to check and confirm these diversities, such as Heterozygosity analysis. 


\section{Contents}

In this tutorial, we will review the steps for performing data analysis using the custom query tools. The custom query tools may be required for analyses that cannot be performed using the existing drop-down options in the form-based query tools available on VivaxGEN. For example, if we are interested in only using samples from certain geographical areas that cannot be selected using the form-based web tools discussed in Tutorial 3. The custom query tools in VivaxGEN are modelled on the NCBI Entrez system, which uses the following syntax:

value [FIELDNAME]

For demonstration purposes, we will use the BTPV batch again for this tutorial. Recall from Tutorial 3 that the samples in the BTPV batch have the following values in the nationality field: india and bhutan. We will review the custom query steps to differentiate the BTPV samples by the nationality field in Principal Coordinate Analysis (PCoA).

\subsection{Using the Custom Query Form}

As a start, let's review the BTPV sample summary after differentiation by the nationality field. Select the Sample summary entry from the Analyze drop-down menu. Next, instead of selecting the batch code as in Tutorial 3, click on the Use query set link just below the Batch code(s) field. The Batch code(s) field will change to a Query set field as illustrated in the following figure.

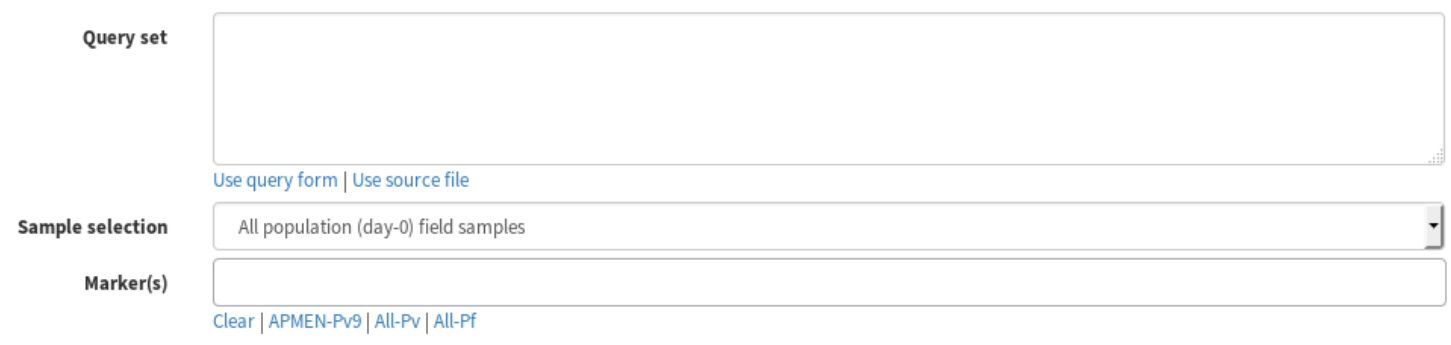

Write or copy the query statement below into the Query set field:

BTPV[batch] !! india[nationality] >> India \$ bhutan[nationality] >> Bhutan

Note, in simple terms, the above query statement essentially makes the following commands:

- use samples from the BTPV batch: "BTPV [batch] “", 
- create a sample set labelled India from the samples that have value india in the nationality field: "india[nationality] >> India"

- create another sample set labelled Bhutan from the samples that have bhutan in the nationality field: "bhutan[nationality] >> Bhutan"

- the double exclamation symbol !! indicates that the former statement will apply to all sample set, so in this case all the sample set must come from BTPV batch

- the dollar sign $\$$ is the sample set separator

- the $>>$ sign indicates the label string

In summary, the above query statement performs re-grouping of the samples in batch BTPV by nationality, enabling comparison of samples from Indian versus Bhutanese nationals.

After adding the query statement, as in Tutorial 3, select the APMEN-Pv9 marker set in the Marker(s) field, and leave the other parameters as default as illustrated in the figure below. Select Execute to perform the analysis.

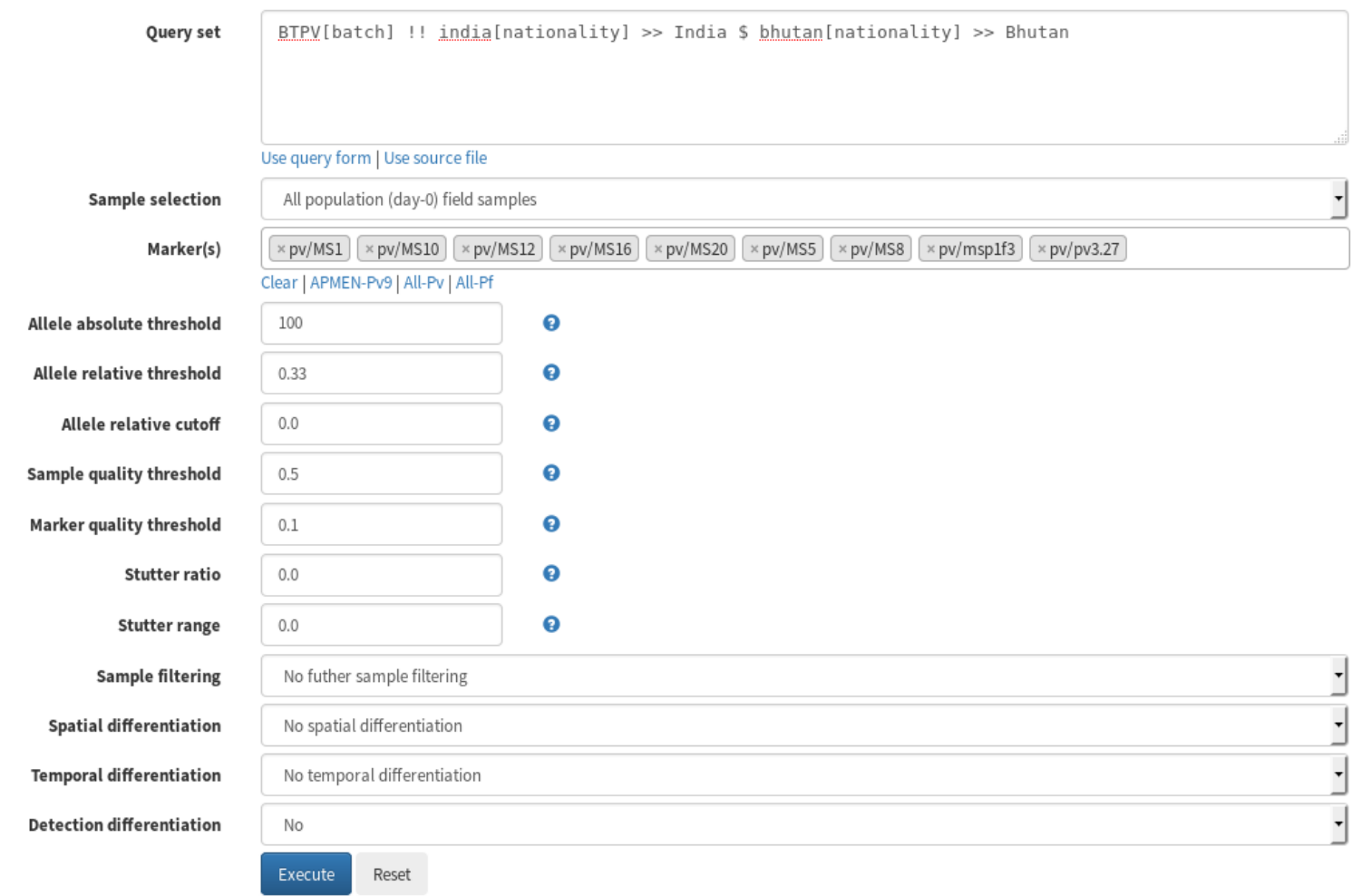

A snapshot of the report outlining the results of the customized sample summary query is illustrated in the figure below. 
Filtering Summary

\begin{tabular}{lcc} 
Label & \multicolumn{1}{l}{ Initial Samples } & Filtered Samples \\
\hline Bhutan & 12 & 12 \\
\hline India & 16 & 16 \\
& & \\
Initial markers & $\mathrm{pv} / \mathrm{MS} 12|\mathrm{pv} / \mathrm{pv} 3.27| \mathrm{pv} / \mathrm{msplf3}|\mathrm{pv} / \mathrm{MS} 10| \mathrm{pv} / \mathrm{MS} 5|\mathrm{pv} / \mathrm{MS} 1| \mathrm{pv} / \mathrm{MS} 8|\mathrm{pv} / \mathrm{MS} 16| \mathrm{pv} / \mathrm{MS} 20:[9]$ \\
Filtered markers & $\mathrm{pv} / \mathrm{MS} 12|\mathrm{pv} / \mathrm{pv} 3.27| \mathrm{pv} / \mathrm{msplf3}|\mathrm{pv} / \mathrm{MS} 10| \mathrm{pv} / \mathrm{MS} 5|\mathrm{pv} / \mathrm{MS} 1| \mathrm{pv} / \mathrm{MS} 8|\mathrm{pv} / \mathrm{MS} 16| \mathrm{pv} / \mathrm{MS} 20:[9]$
\end{tabular}

Bhutan

\begin{tabular}{lc} 
string1 & N \\
\hline passive_detection & N \\
\hline True & 12 \\
\hline symptomatic_status & N \\
\hline False & 12 \\
\hline pcr_identity & N \\
\hline pv & 12 \\
\hline microscopy_identity & \\
\hline pv & N \\
\hline blood_withdrawal & 12 \\
\hline venous & N \\
\hline imported_case & 12 \\
\hline & N \\
\hline
\end{tabular}

As defined in the custom query statement, the results are reported for each of the Indian and Bhutanese nationality sample sets, labelled India and Bhutan respectively.

\subsection{Performing Principal Coordinate Analysis using a Custom Query}

In this step of the tutorial, we will generate a PCoA cluster plot on the BTPV batch with differentiation by nationality. Select the Principle Coordinate Analysis (PCoA) entry from the Analyze drop-down menu. As in step 1, click on the Use query set link, and add the query statement from step 1. Select the APMEN-Pv9 marker set, leave the other parameters as default, and then select Execute.

The results output should provide a PCoA cluster plot with colour-coded differentiation of the samples by nationality as in the figure below. 


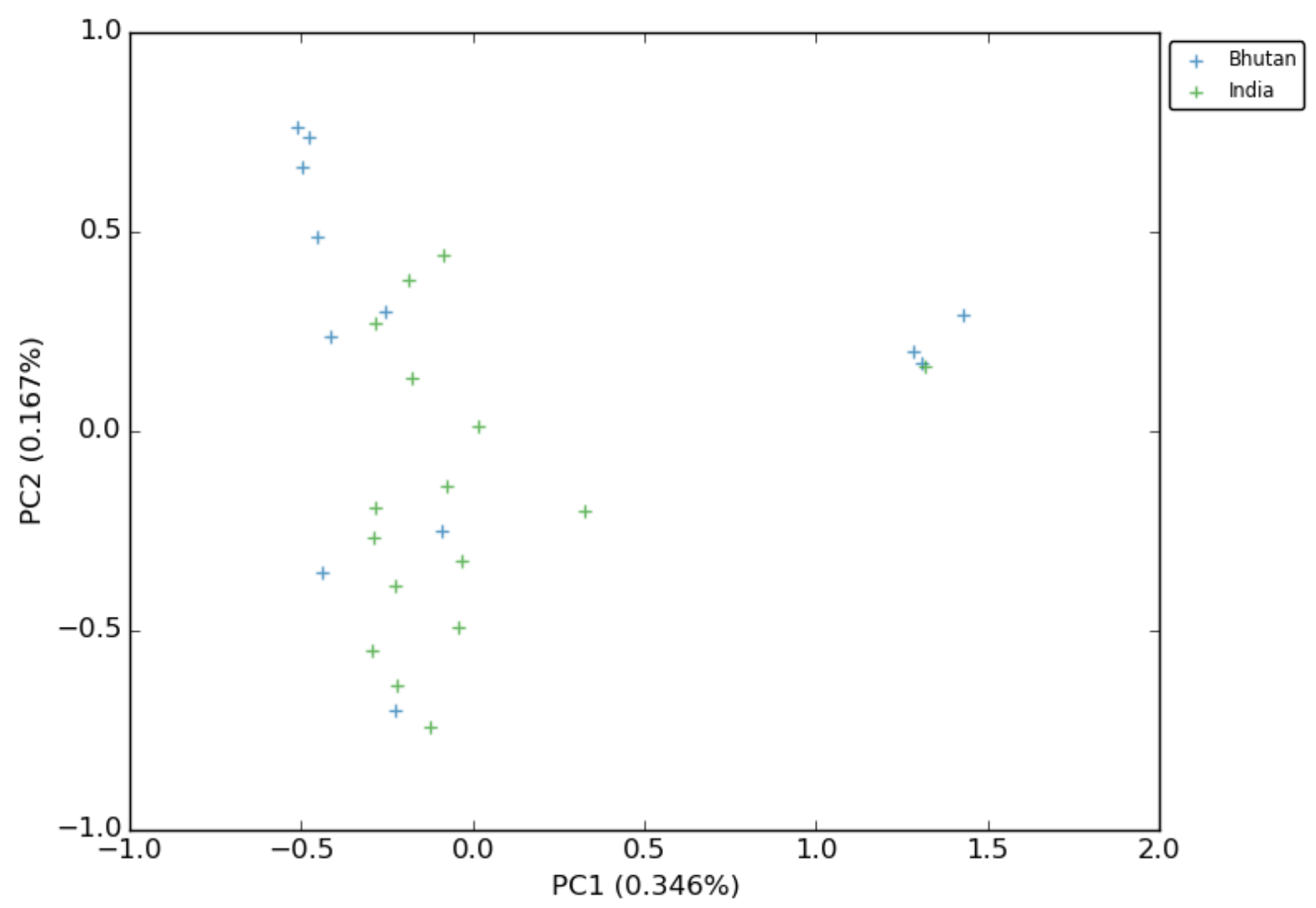




\section{Contents \\ - DATA ANALYSIS WITH YAML FORMAT \\ - A Glance of YAML Format \\ - Using YAML Query \\ - Using YAML Query for Differentiation}

In this tutorial, we will review options for performing data analysis in VivaxGEN using the YAML text format. Note, most users will not need to use the YAML text format - options are provided for more advanced users performing bulk analyses. As stated in the official YAML website, YAML is a human friendly data serialization standard for all programming languages. YAML is a text format that computers can parse and that users can edit and read easily. The YAML format for querying VivaxGEN may be useful to save time where a user needs to perform multiple analyses using different tools with similar queries. In this case, the user can copy and paste the YAML query into the YAML query set, save the query for future use, or for sharing with other users to ensure consistent parameters (and consistent results).

\subsection{A Glance of YAML Format}

An example of YAML format for VivaxGEN is shown below (please note that the indentation of the text is important):

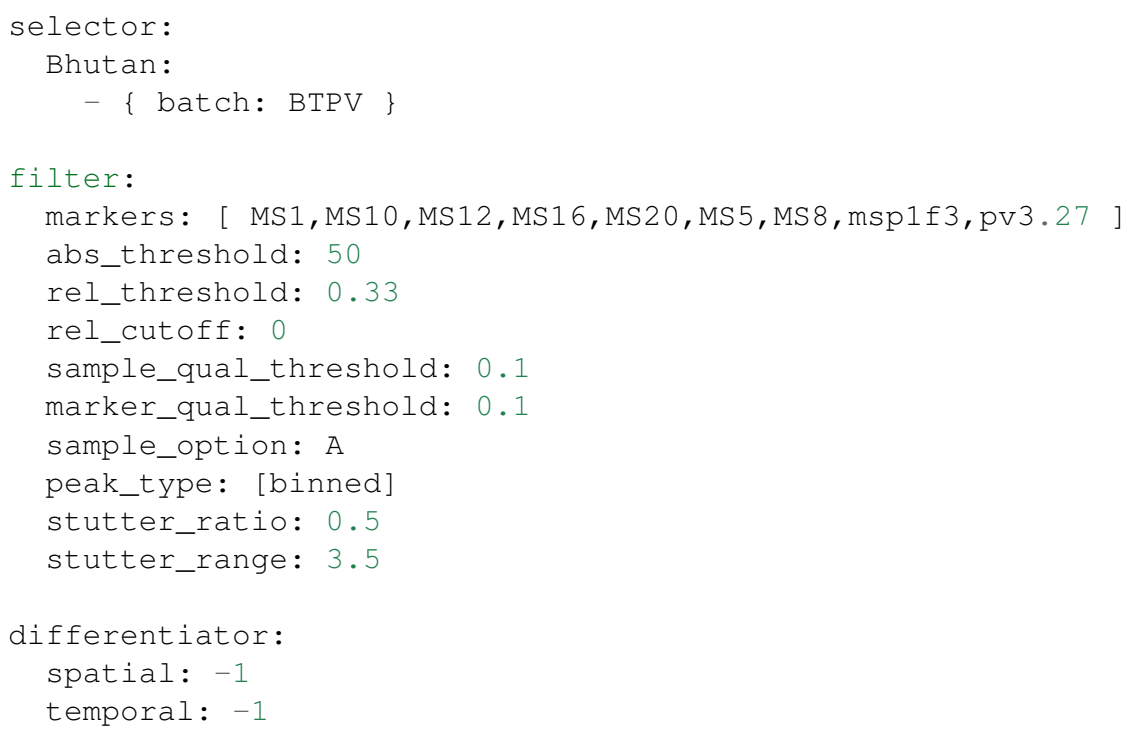

The selector section indicates the sample set that the user wants to create. The above query essentially states that we want to create 1 sample set labelled "Bhutan" which will contain samples from the BTPV batch. 
The filter section controls the parameters used to perform sample, marker and allele filtering. The options are similar to the form-based web fields.

The differentiator section indicates the type of sample differentiation that the user would like to perform on the samples. In the above query, we do not use any differentiation and so we use -1 for all parameter values.

\subsection{Using YAML Query}

To use the YAML format as a query, for example to prepare a Sample summary, we need to click on the YAML Query navigation tab which will change the web form to a simple form as illustrated below.

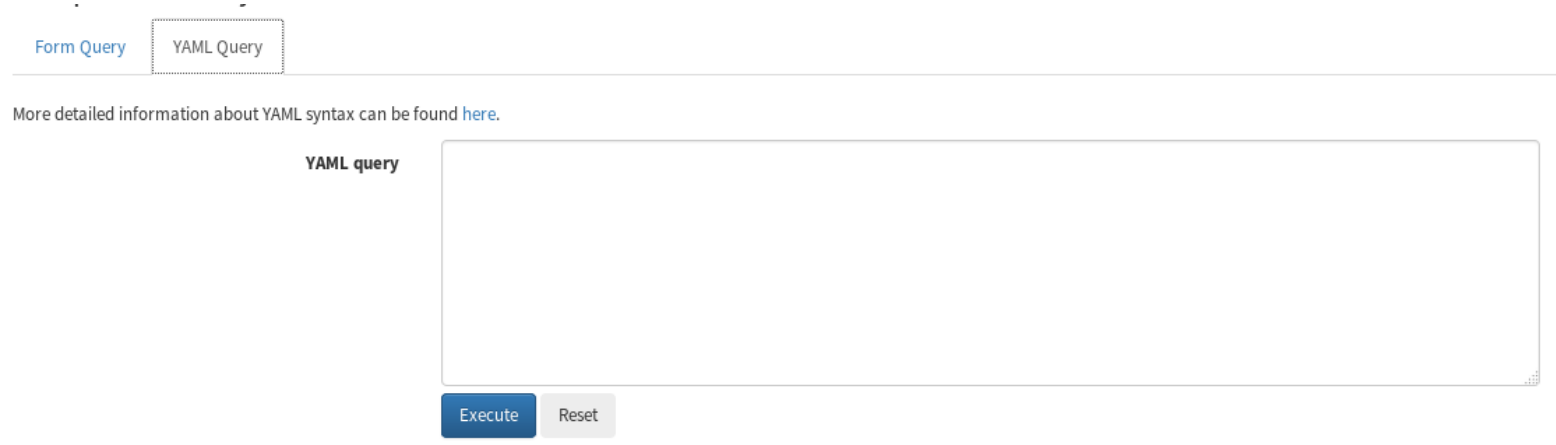

The YAML text then needs to be pasted or directly written into the YAML query field.

For the tutorial, select the Sample summary entry from the Analyze drop-down menu, and paste the above example query into the YAML query field. Select Execute to perform the analysis. The result output should be similar to the output from section 1 in Tutorial 3.

\subsection{Using YAML Query for Differentiation}

Next, we will perform the Principal Coordinate Analysis (PCoA) performed in section 4 of Tutorial 3 using the YAML query format. The YAML query text that we need to use is shown below:

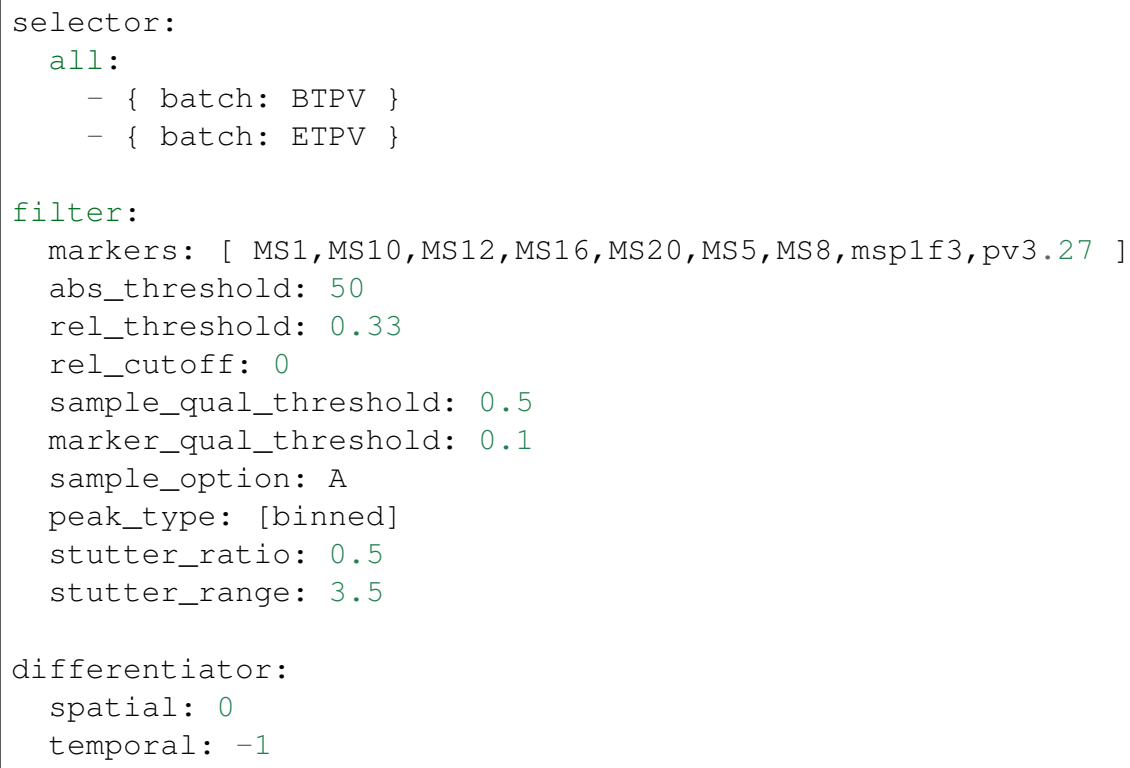

Note that we have defined a single sample sets labelled all, and set spatial in differentiator with value of 0 for country (use 1 for 1 st administrative level, 2 for 2 nd administrative level, etc).

Once we execute the above query, we should get the same result as in section 4 of Tutorial 3 . 
Similary, we can perform the same analysis as in Tutorial 4 i.e. re-grouping the samples by nationality. For this, we need the following query:

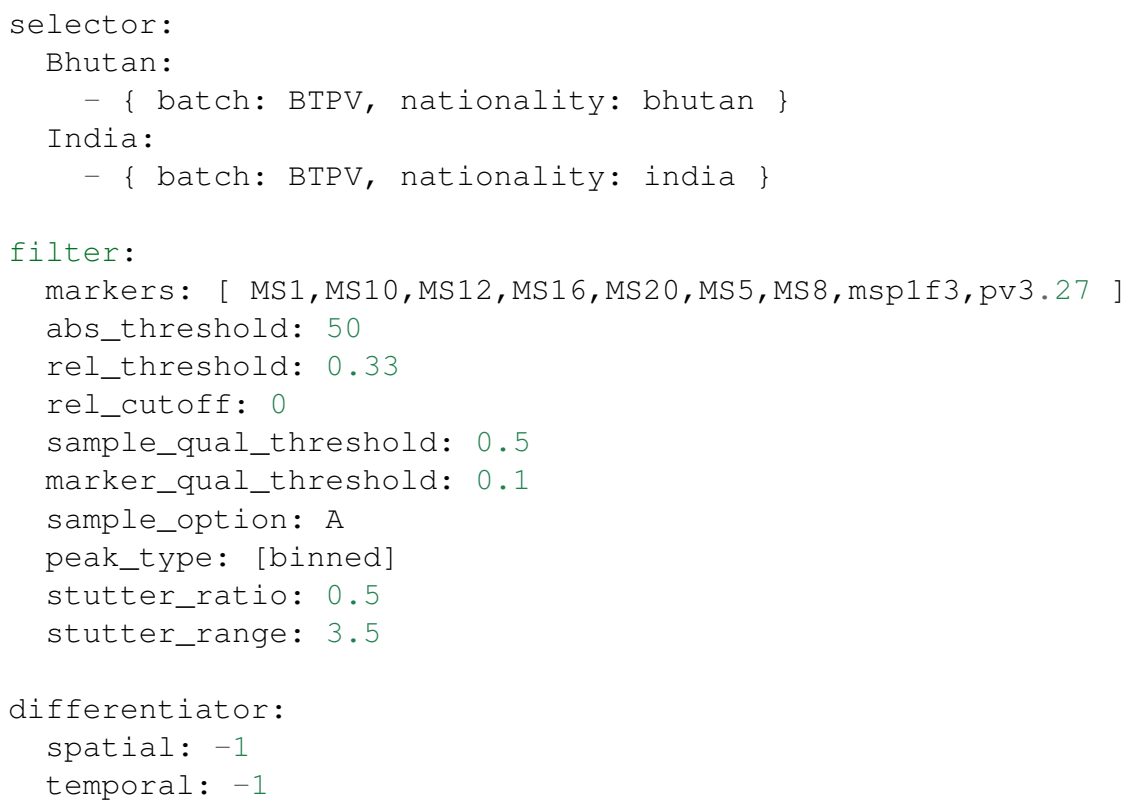

Note that we have defined two sample sets labelled Bhutan and India, with each sample set having a different nationality value but the same batch code.

If we execute the above query in PCoA tool, we should get similar result as in Tutorial 4. Please note that PCoA and Multiple Correspondence Analysis (MCA) will perform data jittering which essentially add small noises to the actual data to prevent to prevent overlap of samples and, hence, different PCoA or MCA plots may look slightly different despite the same data set being applied. 